$\underline{\text { Research Article }}$

\title{
Use of personal protective equipment by healthcare workers exposed to COVID-19 infection at a tertiary care hospital in a lower middle-income country
}

\author{
SK Jayatilleke ${ }^{1}$, DM Mendis ${ }^{1}$,TPW Perera ${ }^{1,2}$, SS Manoj $^{1}$, GK Iresha ${ }^{1}$, \\ PCLS Buddhadasa ${ }^{1}$, HCL Hansani ${ }^{1}$ \\ Sri Lankan Journal of Infectious Diseases 2022Vol.12(1):E6 1-12 \\ DOI: http://dx.doi.org/10.4038/sljid.v12i1.8416
}

\begin{abstract}
Introduction: Data on PPE use and COVID-19 transmission in a healthcare setting is sparse.

Method: This study is a retrospective descriptive study on PPE use and Covid-19 transmission in a hospital. Data collected during routine risk assessment was analyzed using SPSS_26 software.

Results: A total of 108 COVID-19 positive patients were diagnosed in the hospital, of whom 17 were health care workers $(\mathrm{HCW})$. Of the 108, 29 (26.9\%) were asymptomatic. Aerosol generating procedures were performed in 10 events. Fifty three HCWs were quarantined for 14 days following exposure but only 4 developed the disease. Of the total 946 events, 945 had HCWs exposed within 1 metre to COVID-19 positive patients while one event had exposure to the equipment used on a patient. Of the 945 events within a 1 metre distance, attending HCWs wore respirators during $446(47.1 \%)$ events. The source patient wore a mask only in 717 (75.9\%) of the events. In 98 events, exposure was for more than 15 minutes. The $\mathrm{Ct}$ value of the source patient's PCR was between 15.54 to 24.06 or RAT positive when the HCWs acquired the infection while it ranged from 23.28 to 33 when the contacts did not develop the disease. Not having a mask on by the source $(\mathrm{p}=0.014),>15$ minutes exposure within $1 \mathrm{~m}(\mathrm{p}=0.03)$ and not having a face shield in addition to a mask $(\mathrm{p}=0.019)$ had significant association with COVID-19 transmission.
\end{abstract}

Conclusions: Acquiring COVID-19 infection in the hospital setting was uncommon (4/946) in spite of not using respirators during all healthcare exposures. In a quarter of the events, the

${ }^{1}$ Department of Microbiology, Sri Jayewardenepura General Hospital, Sri Lanka

${ }^{2}$ Zhongshan School of Medicine,Sun Yat-sen University, Guangzhou, China

Address for correspondence: Dr. S.K Jayatilleke., 393/C, 3rd Lane, Dutugemunu Mawatha, Battaramulla, Sri Lanka Telephone: +00940714145056 E-mail: kjayatilleke@gmail.com

(iD https://orcid.org/0000-0002-3931-6630

Received 8 September 2021 and revised version accepted 10 December 2021 Published 25.2.22

(c) (1)

This an open-access article distributed under the terms of the Creative Commons Attribution License, which permits unrestricted use, distribution, and reproduction in any medium, provided the original author and source are credited. 
source patients were not wearing a mask. Not having a mask on by the source $(\mathrm{p}=0.014),>15$ minutes exposure within $1 \mathrm{~m}(\mathrm{p}=0.03)$ and not having a face shield in addition to a mask $(\mathrm{p}=0.019)$ had significant association with COVID-19 transmission.

Keywords: COVID-19, healthcare workers, PPE, hospital-acquired, low-middle-income country

\section{Introduction}

Data on the use of personal protective equipment (PPE) and transmission scenarios of COVID19 in healthcare settings is limited globally. This has led to various opinions regarding the use and requirements of different PPE. Sri Jayewardenepura General Hospital (SJGH) is a tertiary care hospital with many specialties and ICU care. During the COVID-19 pandemic, guidelines were prepared and triage areas as well as areas for cohort isolation of patients suspected of COVID-19 were identified to minimize the spread of the disease to healthcare workers and to other patients. A steering committee was appointed, and regular meetings were held to discuss and monitor the issues related to COVID-19 infection. Guidelines for PPE were prepared by the consultant microbiologist (CM) based on the guidelines of the World Health Organization (WHO) and many training sessions were carried out. ${ }^{1}$ However, certain HCWs expressed their dissatisfaction with the PPE recommended by the guidelines and frequently requested higherlevels of PPE that would enable complete body coverage as well as N95 masks when attending to patients. Though the supply from the hospital was most of the time based on the guidelines provided by the CM, some HCWs managed to get other PPE such as N95 masks and use them on a personal basis. A few HCWs did not adhere to the guidelines.

During the study period there was no major community transmission in Sri Lanka, and it was therefore feasible to perform contact tracing. None of the HCWs were vaccinated for COVID-19 during this period as vaccination for COVID-19 was started for HCWs of SJGH from 31.01.2021. Variants of concern were not reported from Sri Lanka during this period.

\section{Objective}

The objective of this study was to evaluate the use of PPE and hospital acquired COVID-19 infection in Sri Jayewardenepura General Hospital (SJGH), Sri Lanka, from $1^{\text {st }}$ October 2020 to $30^{\text {th }}$ January 2021 by HCWs who had contact with COVID-19 positive patients within a $1 \mathrm{~m}$ distance.

Specific objectives were to:

1. describe the exposure of HCWs to COVID-19 along with the PPE used by HCWs in SJGH

2. study the association between PPE use as well as other factors such as patient characteristics and acquisition of COVID-19 by HCWs

\section{Methods}

This was a retrospective descriptive study. HCWs were identified as having high or low risk exposures via data collected at a routine risk assessment following the diagnosis of a COVID-19 positive patient. 
Details were obtained from the data collected on a daily basis by the panel of experts at the risk assessment performed when a patient or a HCW was diagnosed as positive for COVID-19. Most often, such HCWs were contacted either in person or by telephone to collect data related to an exposure.

\section{Definitions:}

- Source patient is a COVID-19 positive patient diagnosed in SJGH with either PCR or Rapid Antigen Test (RAT) who is either a patient who has come for in-patient care or an HCW.

- Events:

○ Being within 1 metre distance to the COVID-19 positive patient, for patient care

○ Being within 1 metre distance for any other reason such as having meals when the patient was another HCW or for cleaning the ward/patient area

- Having contact with equipment used in/on the patient

- Aerosol generating procedure (AGP): The list of AGPs vary in different guidelines. ${ }^{2,3}$ The Northwestern Medicine guidelines were used in this study. ${ }^{3}$

- Healthcare acquired COVID-19: A HCW who tested positive with PCR with or without symptoms within 14 days of documented exposure to a COVID-19 patient in the hospital with no other contact from the community within the 14 days before the date of the positive result.

Table 1 provides definitions of the level of exposure and the hospital policy for management of $\mathrm{HCW}$ with exposure to a COVID-19 positive patient or HCW.

\section{Table 1: Levels of exposures and hospital policy for management}

\begin{tabular}{|l|l|l|}
\hline Exposure level & Description & Hospital policy \\
\hline High risk exposure & $\begin{array}{l}\text { The HCWs who were exposed to a } \\
\text { COVID-19 positive patient within a 1 } \\
\text { metre distance without the } \\
\text { recommended mask }\end{array}$ & $\begin{array}{l}\text { Quarantined the HCW } \\
\text { Monitored for symptoms of COVID-19 } \\
\text { Polymerase Chain Reaction (PCR) test } \\
\text { was performed after 10 days since last } \\
\text { exposure, if remained asymptomatic }\end{array}$ \\
\hline Low risk exposure & $\begin{array}{l}\text { The HCWs who were exposed to a } \\
\text { COVID 19 positive patient within a 1 } \\
\text { metre distance with the recommended } \\
\text { mask but without the recommended } \\
\text { PPE other than the mask. }\end{array}$ & $\begin{array}{l}\text { Allowed to work with the recommended } \\
\text { PPE or quarantined if exposure was for } \\
\text { a long period } \\
\text { Monitored for symptoms of COVID-19 } \\
\text { PCR was performed if they developed } \\
\text { symptoms or after 7 days of exposure if } \\
\text { they remained asymptomatic }\end{array}$ \\
\hline Very low risk exposure & $\begin{array}{l}\text { The HCWs who were taking care of a } \\
\text { COVID-19 positive patient within a 1 } \\
\text { metre distance with the recommended } \\
\text { PPE or those who did not come closer } \\
\text { than 1 metre of the patient. }\end{array}$ & \begin{tabular}{l} 
Neither quarantined nor mandatory PCR \\
\hline
\end{tabular} \\
\hline
\end{tabular}

The associations between having the mask on the source patient, time duration spent near the patient $(<1$ metre), type of mask used, use of face shield and acquiring COVID-19 infection were calculated using SPSS_26 software. 
The association between type of mask used and use of face shield and acquiring COVID-19 infection was also analyzed in the subgroups of events where the source was without the mask and had >15 minutes exposure, using SPSS_26 software.

\section{Results}

During the study period 108 persons were identified as source patients in the hospital. Ninetyone (91) were patients who were admitted to the hospital for care and 17 were healthcare workers. Twenty-nine (29) of the 108 source patients were asymptomatic (26.9\%) while only 26 (24\%) had documented fever. Only $15(11 \%)$ and $23(21.3 \%)$ had shortness of breath and cough respectively. A total of 946 events were recorded during the given period, with $945 \mathrm{HCWs}$ having exposure while within a 1 metre distance from a COVID-19 positive patient and one event where there was exposure to equipment used on a COVID-19 positive patient.

Table 2 provides a summary of characteristics of events where HCWs had exposure to Covid-19 positive patients within a 1 metre distance $(n=945)$.

Table 2: Characteristics of events within a lmetre distance

\begin{tabular}{|c|c|c|c|}
\hline Characteristic of the event & Details of the event & Number & Percentage \\
\hline \multirow[t]{3}{*}{ Source } & Patient & 851 & 90.1 \\
\hline & $\mathrm{HCW}$ & 94 & 9.9 \\
\hline & Total & 945 & \\
\hline \multirow[t]{3}{*}{ Mask on source } & Yes & 717 & 75.9 \\
\hline & No & 228 & 24.1 \\
\hline & Total & 945 & \\
\hline \multirow[t]{4}{*}{ Duration of exposure } & $>15$ minutes & 98 & 10.4 \\
\hline & $5-15$ minutes & 227 & 24.0 \\
\hline & $<5$ minutes & 620 & 65.6 \\
\hline & Total & 945 & \\
\hline \multirow{3}{*}{$\begin{array}{l}\text { PPE status of the exposed } \\
\text { HCW }\end{array}$} & Had at least a mask on & 914 & 96.7 \\
\hline & No PPE (not even a mask) & 31 & 3.3 \\
\hline & Total & 945 & \\
\hline \multirow{3}{*}{$\begin{array}{l}\text { Aerosol generating } \\
\text { procedures performed }\end{array}$} & Yes & 10 & 1.1 \\
\hline & No & 935 & 98.9 \\
\hline & & 945 & \\
\hline \multirow{5}{*}{$\begin{array}{l}\text { Category of HCWs } \\
\text { exposed }\end{array}$} & Nursing officers & 376 & 39.8 \\
\hline & Orderlies & 269 & 28.5 \\
\hline & Medical officers & 194 & 20.5 \\
\hline & Others & 106 & 11.2 \\
\hline & Total & 945 & \\
\hline \multirow{7}{*}{$\begin{array}{l}\text { Different PPE } \\
\text { combinations }\end{array}$} & $\mathrm{N} 95+\mathrm{SM}+\mathrm{FS}+\mathrm{O}+\mathrm{G}$ & 322 & 34.1 \\
\hline & $\mathrm{SM}+\mathrm{FS}+\mathrm{O}+\mathrm{G}$ & 195 & 20.6 \\
\hline & SM only & 125 & 13.2 \\
\hline & $\mathrm{SM}+\mathrm{FS}+\mathrm{O}$ & 70 & 7.4 \\
\hline & Other combinations & 202 & 21.4 \\
\hline & No PPE & 31 & 3.2 \\
\hline & Total & 945 & \\
\hline
\end{tabular}

N95= Respirators (either an N95 mask or an Elastomeric respirator or KN95 mask or an equivalent); $\mathrm{SM}=\mathrm{Medical}$ (Surgical) mask; FS= Face shield; $\mathrm{O}=$ Overall; G-Gloves
The majority (620/945) of exposures were for less than 5 minutes. Only 98 exposures were for more than 15 minutes. AGPs were performed only in 10 events (Table 2).

The source patient was wearing a mask in only 717 of the 945 events $(75.9 \%)$ where there was exposure within 1 metre (Table 2). Surgical masks or respirators were worn in $\quad 914 / 945 \quad(96.7 \%)$ exposure events. In thirtyone events (31/945), the exposed HCW was not wearing any PPE as the source was an $\mathrm{HCW}$. 
Table 3: Events where AGPs were performed (n=10)

\begin{tabular}{|l|l|l|l|l|}
\hline $\begin{array}{l}\text { Category of } \\
\text { HCW }\end{array}$ & Procedure performed & PPE worn & Time duration & $\begin{array}{l}\text { Outcome } \\
\text { (PCR) }\end{array}$ \\
\hline Nursing Officer & Nebulization & SM+G & $>15 \mathrm{~min}$ & COVID +ve \\
\hline Nursing Officer & Nebulization & N95 + G & $<5 \mathrm{~min}$ & COVID -ve \\
\hline Medical Officer & Intubation & N95+SM+FS+O+G & $>15 \mathrm{~min}$ & COVID -ve \\
\hline Nursing Officer & Manual Ventilation & N95+SM+FS+O+G & $>15 \mathrm{~min}$ & COVID -ve \\
\hline Nursing Officer & Manual Ventilation & N95+SM+FS+O+G & $>15 \mathrm{~min}$ & COVID -ve \\
\hline Nursing Officer & $\begin{array}{l}\text { Cardio-Pulmonary } \\
\text { Resuscitation (CPR) }\end{array}$ & N95+SM+FS+O+G & $>15 \mathrm{~min}$ & COVID -ve \\
\hline Nursing Officer & CPR & N95+SM+FS+O+G & $>15 \mathrm{~min}$ & COVID -ve \\
\hline Medical Officer & Intubation & N95+G & $>15 \mathrm{~min}$ & COVID -ve \\
\hline Others & Intubation & N95+O+G & $<5 \mathrm{~min}$ & COVID -ve \\
\hline Nursing Officer & Removed laryngeal tube & SM+FS+O+G & $>15 \mathrm{~min}$ & COVID -ve \\
\hline
\end{tabular}

Fifty-three (53) HCWs were quarantined following exposure for 14 days but only 4 HCWs who were exposed developed disease and/or became PCR positive within the 14 day follow up. They were a nursing officer, two orderlies and a member of the cleaning staff.

Table 4a: Analysis of associated factors with acquiring COVID-19 infection in HCWs with exposure within 1 metre $(n=945)$

\begin{tabular}{|c|c|c|c|c|}
\hline \multicolumn{2}{|l|}{ Associated factor } & $\begin{array}{c}\text { Disease } \\
\text { transmitted }\end{array}$ & $\begin{array}{l}\text { No disease } \\
\text { transmission }\end{array}$ & $P$ value \\
\hline \multirow[t]{2}{*}{ Duration of exposure } & $<15$ mins & 1 & 846 & \multirow[t]{2}{*}{0.030} \\
\hline & $>15$ mins & 2 & 96 & \\
\hline \multirow[t]{2}{*}{ Performing an AGP } & Yes & 1 & 9 & \multirow[t]{2}{*}{0.031} \\
\hline & No & 2 & 933 & \\
\hline \multirow[t]{2}{*}{ Mask on source } & With mask & 0 & 717 & \multirow[t]{2}{*}{0.014} \\
\hline & No mask & 3 & 225 & \\
\hline \multirow{2}{*}{$\begin{array}{l}\text { Exposed HCW wearing } \\
\text { any mask }\end{array}$} & Yes & 3 & 911 & \multirow[t]{2}{*}{1.000} \\
\hline & No & 0 & 31 & \\
\hline \multirow{2}{*}{$\begin{array}{l}\text { Exposed HCW wearing } \\
\text { a respirator }\end{array}$} & Yes & 0 & 445 & \multirow[t]{2}{*}{0.252} \\
\hline & No & 3 & 497 & \\
\hline \multirow{2}{*}{$\begin{array}{l}\text { Exposed HCW wearing } \\
\text { face-shield (in addition } \\
\text { to the mask) }\end{array}$} & Yes & 0 & 694 & \multirow[t]{2}{*}{0.019} \\
\hline & No & 3 & 248 & \\
\hline
\end{tabular}

More than 15 minutes of exposure, performing an aerosol generating procedure and not having a mask on the source patient were significantly associated with HCWs working within a 1metre distance acquiring COVID-19 infection. Not wearing a face shield in addition to other PPE was significantly associated with acquiring COVID-19 with $\mathrm{p}$ value $<0.05$ in all those who worked within a 1 metre distance as well as in the group who worked close to patients without a mask. (Table 4a-d 
Table 4b: Analysis of factors associated with acquiring COVID-19 infection in HCWs who had exposure to a COVID-19 patient within 1m for $>15$ minutes $(n=98)$

\begin{tabular}{|c|c|c|c|c|}
\hline \multicolumn{2}{|l|}{ Associated factor } & \multirow{2}{*}{$\begin{array}{c}\text { Disease } \\
\text { transmitted } \\
1\end{array}$} & \multirow{2}{*}{$\begin{array}{c}\text { No disease } \\
\text { transmission }\end{array}$} & \multirow{2}{*}{$\begin{array}{c}\text { P value } \\
0.138 \\
\end{array}$} \\
\hline Performing an AGP & Yes & & & \\
\hline & No & 1 & 90 & \\
\hline \multirow[t]{2}{*}{ Mask on source } & With mask & 0 & 52 & \multirow[t]{2}{*}{0.218} \\
\hline & No mask & 2 & 44 & \\
\hline \multirow{2}{*}{$\begin{array}{l}\text { Exposed HCW wearing } \\
\text { a respirator }\end{array}$} & Yes & 0 & 42 & \multirow[t]{2}{*}{0.505} \\
\hline & No & 2 & 54 & \\
\hline \multirow{2}{*}{$\begin{array}{l}\text { Exposed HCW wearing } \\
\text { face-shield (in addition } \\
\text { to the mask) }\end{array}$} & Yes & 0 & 53 & \multirow[t]{2}{*}{0.208} \\
\hline & No & 2 & 43 & \\
\hline
\end{tabular}

Table 4c: Analysis of factors associated with acquiring COVID-19 infection in HCWs with exposure to a COVID-19 patient who were not wearing a mask at the time of exposure $(n=228)$

\begin{tabular}{|c|c|c|c|c|}
\hline \multicolumn{2}{|l|}{ Associated factor } & \multirow{2}{*}{$\begin{array}{c}\begin{array}{c}\text { Disease } \\
\text { transmitted }\end{array} \\
0\end{array}$} & \multirow{2}{*}{$\begin{array}{c}\begin{array}{c}\text { No disease } \\
\text { transmission }\end{array} \\
104 \\
\end{array}$} & \multirow{2}{*}{$\begin{array}{c}\mathrm{P} \text { value } \\
0.252\end{array}$} \\
\hline Exposed HCW wearing & Yes & & & \\
\hline a respirator & No & 3 & 121 & \\
\hline \multirow{2}{*}{$\begin{array}{l}\text { Exposed HCW wearing } \\
\text { face-shield (in addition } \\
\text { to the mask) }\end{array}$} & Yes & 0 & 143 & \multirow[t]{2}{*}{0.051} \\
\hline & No & 3 & 82 & \\
\hline
\end{tabular}

Table 4d: Analysis of factors associated with acquiring COVID-19 infection in HCWs who had exposure to a COVID-19 patient while performing AGPs $(n=10)$

\begin{tabular}{|c|c|c|c|c|}
\hline \multicolumn{2}{|l|}{ Associated factor } & $\begin{array}{c}\text { Disease } \\
\text { transmitted }\end{array}$ & $\begin{array}{l}\text { No disease } \\
\text { transmission }\end{array}$ & $\mathrm{P}$ value \\
\hline \multirow{2}{*}{$\begin{array}{l}\text { Exposed HCW wearing } \\
\text { a respirator }\end{array}$} & Yes & 0 & 8 & \multirow[t]{2}{*}{0.200} \\
\hline & No & 1 & 1 & \\
\hline \multirow{2}{*}{$\begin{array}{l}\text { Exposed HCW wearing } \\
\text { face-shield (in addition } \\
\text { to the mask) }\end{array}$} & Yes & 0 & 7 & \multirow[t]{2}{*}{0.300} \\
\hline & No & 1 & 2 & \\
\hline
\end{tabular}


Table 5: Events where no PPE were worn $(n=31)$

\begin{tabular}{|c|c|c|c|c|c|c|c|c|}
\hline \multirow{2}{*}{$\begin{array}{c}\text { Category of } \\
\text { HCW }\end{array}$} & \multirow{2}{*}{$\begin{array}{l}\text { Description } \\
\text { of contact } \\
\text { event }\end{array}$} & \multirow{2}{*}{$\begin{array}{c}\text { Time } \\
\text { duration }\end{array}$} & \multicolumn{4}{|c|}{ Source } & \multirow{2}{*}{$\begin{array}{c}\text { Outcome } \\
\text { (COVID } \\
\text { positive } \\
\text { or } \\
\text { negative) }\end{array}$} & \multirow{2}{*}{$\begin{array}{l}\text { AB test } \\
\text { result for } \\
\text { SARS- } \\
\text { CoV-19 } \\
2,4 \text { weeks } \\
\text { following } \\
\text { exposure }\end{array}$} \\
\hline & & & Category & $\begin{array}{l}\mathrm{Ct} / \\
\mathrm{RAT} \\
+\mathrm{ve}\end{array}$ & $\begin{array}{c}\text { Symptomatic } \\
\text { or not }\end{array}$ & $\begin{array}{l}\text { Mask } \\
\text { on } \\
\text { source }\end{array}$ & & \\
\hline $\begin{array}{l}\text { Medical } \\
\text { Officer }\end{array}$ & Having tea & $10 \mathrm{~min}$ & $\begin{array}{l}\text { Nursing } \\
\text { Officer }\end{array}$ & $\begin{array}{l}S=25.5 \\
E=26.6\end{array}$ & $\begin{array}{l}\text { Symptomatic } \\
\text { (sore throat, } \\
\text { body aches) }\end{array}$ & No & Negative & Not done \\
\hline Orderly & $\begin{array}{l}\text { Talked with } \\
\text { the patient }\end{array}$ & $10 \mathrm{~min}$ & & & & & & \\
\hline $\begin{array}{c}6 \\
\text { Management } \\
\text { assistants } \\
\end{array}$ & Had tea & $>15 \mathrm{~min}$ & $\begin{array}{l}\text { Management } \\
\text { assistant }\end{array}$ & $\begin{array}{l}S=27.9 \\
E=28.01\end{array}$ & $\begin{array}{l}\text { Symptomatic } \\
\text { (fever, } \\
\text { coryza) }\end{array}$ & Yes & Negative & Not done \\
\hline 5 Orderlies & $\begin{array}{l}\text { Had tea } \\
\text { with Pt }\end{array}$ & $<5 \min$ & Orderly & $\begin{array}{l}S=24.2 \\
E=24.9\end{array}$ & Symptomatic & No & Negative & Not done \\
\hline 1 Orderly & $\begin{array}{l}\text { Lunch chat } \\
\text { with +ve pt. }\end{array}$ & $<5 \min$ & & & & & & \\
\hline 1 Orderly & $\begin{array}{l}\text { Had tea } \\
\text { (shared the } \\
\text { cup) }\end{array}$ & $<5 \min$ & & & & & & \\
\hline $\begin{array}{l}\text { Nursing } \\
\text { officer }\end{array}$ & Talk with pt & $<5 \min$ & & & & & & \\
\hline 2 Orderlies & $\begin{array}{l}\text { Lunch chat } \\
\text { with + ve pt. }\end{array}$ & $5-15 \mathrm{~min}$ & & & & & & \\
\hline 3 Orderlies & $\begin{array}{l}\text { Lunch chat } \\
\text { with +ve pt. }\end{array}$ & $>15 \mathrm{~min}$ & & & & & & \\
\hline $\begin{array}{l}2 \\
\text { Management } \\
\text { assistants }\end{array}$ & Had lunch & $>30 \mathrm{~min}$ & $\begin{array}{l}\text { Management } \\
\text { assistant }\end{array}$ & $\begin{array}{l}S=28.13 \\
E=28.45\end{array}$ & $\begin{array}{l}\text { Symptomatic } \\
\text { (coryza) }\end{array}$ & No & Negative & Negative \\
\hline $\begin{array}{l}\text { Orderly } \\
\text { supervisor }\end{array}$ & $\begin{array}{l}\text { Visited } \\
\text { home }\end{array}$ & $>30 \mathrm{~min}$ & & & & & & \\
\hline Orderly & $\begin{array}{l}\text { Talked with } \\
\text { the patient }\end{array}$ & $<5 \min$ & Orderly & $\begin{array}{l}S=23.66 \\
E=23.28\end{array}$ & $\begin{array}{l}\text { Symptomatic } \\
\text { (fever, cough, } \\
\text { nausea, } \\
\text { vomiting, } \\
\text { coryza) }\end{array}$ & Yes & Negative & Negative \\
\hline 6 Doctors & $\begin{array}{l}\text { Had tea } \\
\text { with patient }\end{array}$ & $>15 \mathrm{mts}$ & Doctor & $\begin{array}{l}S=33 \\
E=33\end{array}$ & $\begin{array}{l}\text { Symptomatic } \\
\text { (fever, sore } \\
\text { throat, } \\
\text { headache) }\end{array}$ & No & Negative & Not done \\
\hline
\end{tabular}

$\mathrm{AB}$ - antibody; pt. - patient

Four healthcare workers developed healthcare acquired COVID-19. Three were exposed while working within the one metre distance from the patient and the other HCW while washing the equipment used on the patient. Details of the exposures are given in Table 6 
Table 6: Exposure of $4 \mathrm{HCWs}$ who acquired health care associated COVID-19

\begin{tabular}{|c|c|c|c|c|}
\hline $\begin{array}{l}\text { Category } \\
\text { of HCW }\end{array}$ & Patient status & Mask on source & $\begin{array}{c}\text { PPE worn by HCW/time } \\
\text { of exposure }\end{array}$ & Procedure performed \\
\hline $\begin{array}{l}\text { Nursing } \\
\text { Officer }\end{array}$ & $\begin{array}{l}44 \text { yr old patient } \\
4 \text { years post kidney } \\
\text { transplant presented with } \\
\text { fever and shortness of } \\
\text { breath } \\
\text { Patient was restless } \\
\text { RT PCR positive } \\
\text { Ct value of S } 15.54 ; \mathrm{E} \\
\text { gene } 15.55\end{array}$ & $\begin{array}{l}\text { not wearing the } \\
\text { mask as he was } \\
\text { short of breath }\end{array}$ & $\begin{array}{l}\text { overall, surgical mask } \\
\text { and face shield. } \\
\text { Nurse had to remove face } \\
\text { shield as visibility was } \\
\text { poor and she was finding } \\
\text { it difficult to insert a } \\
\text { cannula. / } 45 \text { minutes } \\
\text { Did not change the mask } \\
\text { until the shift was over } \\
\text { (during the night shift). }\end{array}$ & $\begin{array}{l}\text { Inserted an intravenous } \\
\text { cannula and nebulized the } \\
\text { patient }\end{array}$ \\
\hline $\begin{array}{l}\text { Cleaning } \\
\text { staff } \\
\text { member }\end{array}$ & $\begin{array}{l}\text { 27year old boy } \\
\text { RAT positive }\end{array}$ & $\begin{array}{l}\text { abdominal pain } \\
\text { and was not } \\
\text { wearing the mask }\end{array}$ & $\begin{array}{l}\text { Only surgical mask / } \\
\text { Around } 5 \text { mins within } 1 \\
\text { meter }\end{array}$ & $\begin{array}{l}\text { No procedure on patient } \\
\text { HCW swept the ward with } \\
\text { a broom. }\end{array}$ \\
\hline $\begin{array}{l}\text { Orderly } \\
2 \text { events }\end{array}$ & $\begin{array}{l}\text { 56yr old patient - known } \\
\text { diabetic with anaemia } \\
\text { and abdominal pain } \\
\text { admitted for } \\
\text { colonoscopy. } \\
\text { RAT was positive. } \\
\text { RT PCR positive } \\
\text { Ct value of S-20.33; E } \\
\text { gene-18.33 }\end{array}$ & $\begin{array}{l}\text { Event 1: Patient } \\
\text { not wearing mask } \\
\text { (she was brushing } \\
\text { her teeth) } \\
\text { Event 2: Patient } \\
\text { was not wearing a } \\
\text { mask. }\end{array}$ & $\begin{array}{l}\text { Event 1: Overall and the } \\
\text { surgical mask only }-15 \\
\text { mins } \\
\text { Event 2: Surgical mask } \\
\text { only - }<5 \text { mins }\end{array}$ & $\begin{array}{l}\text { No procedure on patient } \\
\text { Event 1: HCW helped } \\
\text { another patient to wash the } \\
\text { face while the COVID } \\
\text { positive patient was } \\
\text { brushing her teeth in a } \\
\text { nearby sink in the wash } \\
\text { room- considered as } \\
\text { significant } \\
\text { Event } 2 \text { : flask was filled } \\
\text { and given to the patient } \\
\text { drink }\end{array}$ \\
\hline $\begin{array}{l}\text { Orderly } \\
2 \text { events } \\
\text { on same } \\
\text { day }\end{array}$ & $\begin{array}{l}41 \text { yr old male patient } \\
\text { who underwent bladder } \\
\text { resection and biopsy. } \\
\text { RAT was negative. } \\
\text { RT PCR positive } \\
\text { Ct value of S-23.29; E } \\
\text { gene-24.06. }\end{array}$ & $\begin{array}{l}\text { Event 1: not } \\
\text { relevant }\end{array}$ & $\begin{array}{l}\text { Event 1: Overall and the } \\
\text { N95 mask only/15 mins }\end{array}$ & $\begin{array}{l}\text { No procedures on patient } \\
\text { Event 1: Washed the } \\
\text { equipment including the } \\
\text { laryngoscope blade used } \\
\text { on patient in the theatre - } \\
\text { considered as significant } \\
\text { Event } 2 \text { : Transported the } \\
\text { patient on the trolley to the } \\
\text { theatre and back from the } \\
\text { reception }\end{array}$ \\
\hline
\end{tabular}

RT PCR (RealStar® SARS-CoV-2 RT-PCR Kit 1.0, Altona Diagnostics, Germany).

RAT - Rapid antigen test 


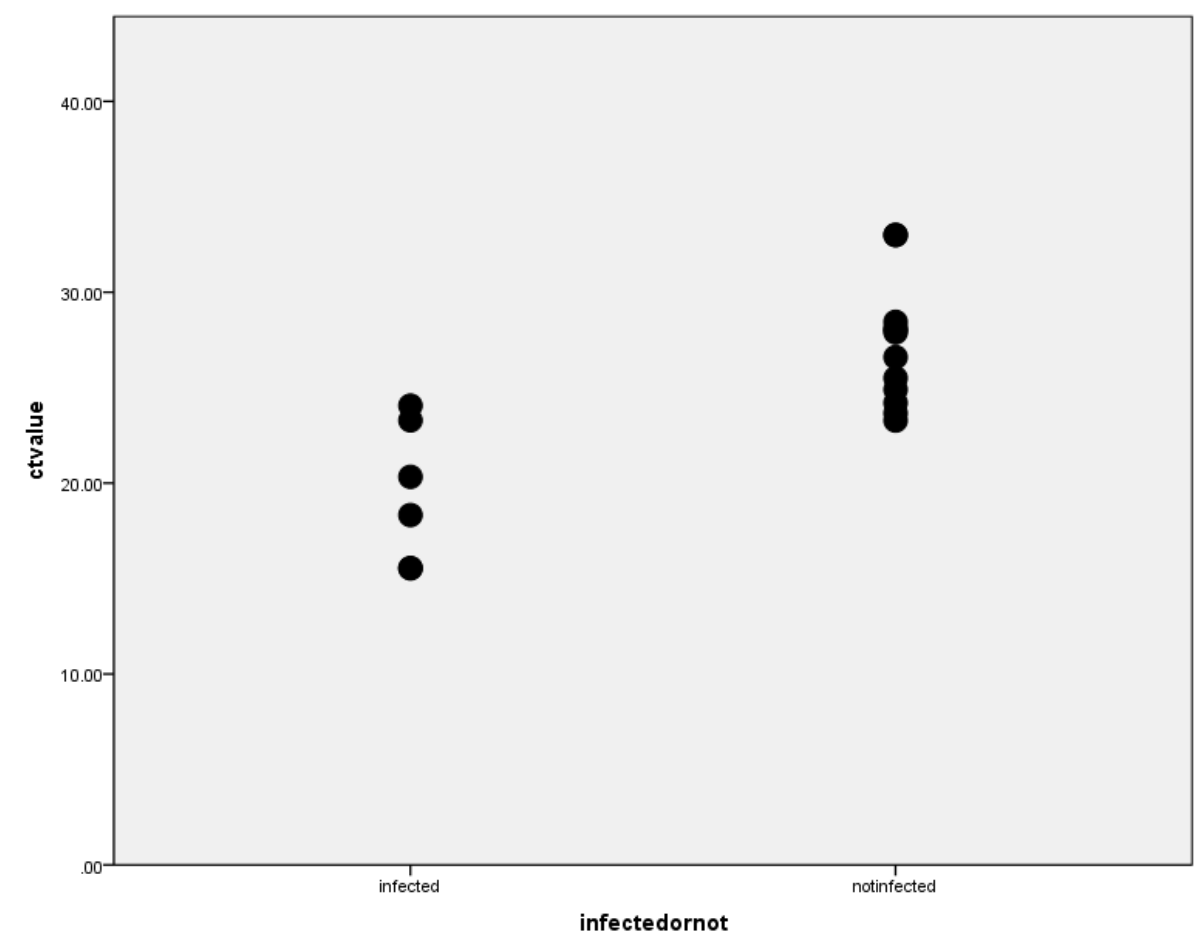

Figure 1: Cycle threshold in PCR of source patients

Figure 1 demonstrates the cycle threshold in PCR of the source patients who infected HCWs and who did not infect HCWs but were not wearing PPE. The $\mathrm{Ct}$ values of the source patients who had infected HCWs ranged from 15.54 to 24.06 (mean=19.5; SD=3.38) while patients who did not have secondary cases in spite of not having any PPE on (Table 5) had $\mathrm{Ct}$ values ranging from 23.28 to 33 (mean=27.3; $\mathrm{SD}=3.2$ ). (Figure 1 )

\section{DISCUSSION}

In Sri Lanka, though there were specially identified hospitals for management of patients with COVID-19 disease, during the study period a significant number of patients were looked after for a short period in Sri Jayewardenepura General Hospital. During this period, as there was no widespread transmission of COVID-19 in the community, contact tracing was feasible in a reliable manner. Many healthcare workers acquired the disease from their household contacts and other contacts outside the hospital. All patients as well as HCWs who had one or more symptoms suggestive of COVID-19 were tested with PCR or RAT. The patients who were admitted for surgery and other procedures were also subjected to PCR or RAT.

One hundred and eight COVID-19 positive patients were identified in the hospital during the study period. Twenty nine of them were asymptomatic while only a minority had typical 
symptoms such as cough and shortness of breath. This is comparable to the meta-analysis which reported that asymptomatic cases could account for more than $20 \%$ of all COVID-19 patients. ${ }^{4}$

Nearly a quarter of the source patients were not wearing the mask (Table 2). This was probably due to their symptoms such as shortness of breath and the therapeutic needs such as oxygen therapy in most patients. This is important to be considered when deciding on guidelines in healthcare settings as it is not practical to have the mask always on patients, especially when they are ill.

Nursing officers had the highest number of exposures within the 1metre distance followed by the orderlies (Table 2).

The significant exposure of $>15$ minutes were in only 98 events. Aerosol generating procedures were performed only in a few events $(n=10)$. Whether this was due to fear of COVID-19 disease has to be considered. This may have delayed the optimum treatment in patients.

In the current study, intubation, manual ventilation (Ambu-bag resuscitation), cardio-pulmonary resuscitation (CPR), removal of laryngeal tube and nebulization were possible AGPs encountered (Table 3). One of the $2 \mathrm{HCWs}$ who were involved with AGP without N95, or equivalent masks got infected within the 14 day follow up period. She was a nursing officer who had nebulized the patient for nearly 45 minutes.

The numbers of HCWs infected were only 4 in spite of having 946 exposure events to 108 patients. There are few publications on healthcare acquired COVID-19 available for comparison. A systematic review concluded that infections and deaths among HCWs follow that of the general population around the world ${ }^{5}$ and in others, a high rate of infection among HCWs were reported. ${ }^{6,7}$ More than 15 minutes of exposure, performing an aerosol generating procedure and not having a mask on the source patient were significantly associated with acquiring COVID-19 infection (Table 4). WHO and local guidelines on PPE had considered AGPs as high risk procedures.

Not wearing a mask and not wearing an N95 or equivalent mask by the contact HCW did not have a significant association with acquiring COVID-19 infection but not wearing a face shield in addition to a mask did have a significant association with acquiring COVID-19 infection (Table 4). This may be because the masks were not worn in the correct way to cover the nose and the mouth by the HCWs. The fit test is not performed for N95 and equivalent masks and most of the available masks were loose fitting for the HCWs. Wearing the face shield in addition to the mask and other PPE had a significant association with reduction of acquiring infection in all those who had exposure within the 1 metre distance as well as when the source was not wearing a mask (Table 4). None of the HCWs were wearing the face-shield without a mask.

Analysis of associated factors in groups who had >15-minute exposure, and AGP performing events did not show a significant association with any PPE use, probably due to the small number of events (Table 4). 
Though there were 98 events where HCWs had exposure to a COVID-19 positive patient for $>15$ minutes, only 2 of them became positive for COVID-19 within the 14-day follow-up period. Of the 98 events, N95 or equivalent masks were worn only in 42 (42.9\%) of the events. None of the HCWs were vaccinated for COVID-19 infection during this period and the limited number of antibody tests done on the exposed HCWs after 14 days of exposure were not positive, indicating they were not protected by antibodies. During the study period, none of the COVID-19 variants of concern were identified in Sri Lanka.

Of the 31 events where HCWs were exposed to a COVID-19 positive HCW without any PPE, Ct values of the PCR in the source HCWs who were symptomatic at the time of exposure ranged from 23.28 to 33. None of the exposed HCWs got the disease. This is probably due to the high $\mathrm{Ct}$ values of the source patient indicating low viral load. On the other hand, the source patients of the events in which HCWs were infected had either a RAT positive or a PCR positive with a $\mathrm{Ct}$ value of less than 25 (Chart 1).

In HCWs who became positive during the 14-day follow-up period, only one (in event number 1) was providing direct care to the patient. Washing the equipment of patient, being near the patient when she was brushing her teeth and cleaning the patient's ward area were the other factors which probably led to acquiring infection.

\section{Conclusion}

Wearing a mask is useful for source control and therefore should be encouraged as much as possible. However, wearing a mask may not be practical, especially if the patient has symptoms of COVID-19, limiting the usefulness of the mask for source control in the healthcare setting. Acquiring COVID-19 infection in hospital setting was not common in spite of not using respirators during all healthcare encounters. HCWs acquired infection when the source patients had Ct values of less than 25 in the PCR or were RAT positive. More than 15 minutes of exposure, performing an aerosol generating procedure and not having a mask on the source patient were significantly associated with acquiring COVID-19 infection. Wearing the faceshield in addition to a mask was significantly associated with not acquiring COVID-19 in exposed HCWs.

Limitations: Retrospective data collection and small number of patients are the limitation of this study.

\section{Declarations}

\footnotetext{
Acknowledgements: I would like to acknowledge all HCWs who participated in this study by providing data

Funding source: None.

Conflict of Interest statement: The authors declare that there are no conflicting interests.

Ethics statement: Ethical approval taken from the ERC of SJGH

Author contributions:

S.K. Jayatilleke- Design of study, experimental work, analysis of data and writing manuscript

T. P. W. Perera- Experimental work and analysis of data

D. M. Mendis, S. S. Manoj, G. K. Iresha, P. C. L. S. Buddhadasa and H. C. L. Hansani- Experimental work
} 


\section{References}

1. Rational use of personal protective equipment for COVID-19 and considerations during severe shortages: Interim guidance; 23 December 2020; World Health Organization; https://www.who.int/publications/i/item/rational-use-of-personal-protective-equipment-forcoronavirus-disease-(covid-19)-and-considerations-during-severe-shortages

2. Michael Klompas, MeghanBaker, ChanuRhee. What is an aerosol-generating procedure? JAMA Surg. 2021;156(2):113-114.; doi: 10.1001/jamasurg.2020.6643

3. Aerosol-Generating Procedures (AGPs): Note: This information is subject to change based on new research and evidence; Northwestern Medicine; Effective May 4, 2021 https://physicianforum.nm.org/uploads/1/1/9/4/119404942/what_are_aerosolgenerating_procedures_or_agps.pdf

4. Andreas Kronbichler, Daniela Kresse, Sojung Yoon, et al. Asymptomatic patients as a source of COVID-19 infections: A systematic review and meta-analysis. Int J Infect Dis. 2020; 98:180-186; doi: 0.1016/j.ijid.2020.06.052

5. Bandyopadhyay S, Baticulon RE, Kadhum M, et al. Infection and mortality of healthcare workers worldwide from COVID-19: a systematic review. BMJ Global Health 2020; 5:e003097. doi: http://dx.doi.org/10.1136/ bmjgh-2020

6. Lotta-maria A.H. Oksanen, EnniSanmark, et al. Sources of healthcare workers' COVID-19 infections and related safety guidelines.International Journal of Occupational Medicine and Environmental Health 2021;34(2):239-249; doi: https://doi.org/10.13075/ijomeh.1896.01741

7. MandanaGholamia, Iman Fawada, Sidra Shadana et al. COVID-19 and healthcare workers: A systematic review and meta-analysis; International Journal of Infectious Diseases 2021; 104:335-346 doi: https://doi.org/10.1016/j.ijid.2021.01.013 